\title{
Associations between serum phthalates and biomarkers of reproductive function in 589 adult men
}

\author{
Ina Olmer Specht ${ }^{\mathrm{a}, *}$, Gunnar Toft ${ }^{\mathrm{b}}$, Karin S. Hougaard ${ }^{\mathrm{c}}$, Christian H. Lindh ${ }^{\mathrm{d}}$, Virissa Lenters ${ }^{\mathrm{e}}$, Bo A.G. Jönsson ${ }^{\mathrm{d}}$, \\ Dick Heederik $^{\mathrm{e}}$, Aleksander Giwercman ${ }^{\mathrm{f}}$, Jens Peter E. Bonde ${ }^{\mathrm{a}}$
}

a Department of Occupational and Environmental Medicine, University Hospital of Copenhagen, DK-2400 Copenhagen NV, Denmark

${ }^{\mathrm{b}}$ Department of Occupational Medicine, Aarhus University Hospital, DK-8000 Aarhus C, Denmark

c National Research Centre for the Working Environment, DK-2100 Copenhagen Ø, Denmark

${ }^{\mathrm{d}}$ Division of Occupational and Environmental Medicine, Lund University, SE-221 85 Lund, Sweden

e Division of Environmental Epidemiology, Institute for Risk Assessment Sciences, Utrecht University, NL-3584 CM Utrecht, The Netherlands

${ }^{\mathrm{f}}$ Reproductive Medicine Centre, Malmö University Hospital, Lund University, Malmö SE-20502, Sweden

\section{A R T I C L E I N F O}

\section{Article history:}

Received 11 November 2013

Accepted 6 February 2014

Available online 26 February 2014

\section{Keywords:}

Di-2-ethylhexyl phthalate

Diisononyl phthalate

Fertility

Reproduction

Metabolic pathway

Testosterone

\begin{abstract}
A B S T R A C T
Phthalates which are widely used, are ubiquitous in the environment and in some human tissues. It is generally accepted that phthalates exert their toxic action by inhibiting Leydig cell synthesis of testosterone, but in vitro studies have also shown anti-androgenic effects at the receptor level. Some cross-sectional studies have shown inverse associations between urinary levels of phthalates and reproductive hormones, but results are conflicting and the evidence base is limited. The aim of this study was to investigate if levels of di-2-ethylhexyl phthalate (DEHP) and diisononyl phthalate (DiNP) metabolites in serum are associated with serum concentrations of male reproductive hormones and semen quality. A secondary aim was to investigate metabolic pathways of DEHP and DiNP on semen quality and reproductive hormones.

A cross-sectional sample of 589 spouses of pregnant women from Greenland, Poland and Ukraine were enrolled between 2002 and 2004. The men gave semen and blood samples and were interviewed. Six phthalate metabolites of DEHP and DiNP were measured by liquid chromatography tandem mass spectrometry in serum. The metabolites were summed according to their molar weight.

We observed significant inverse associations between serum levels of the metabolites, the proxies and serum testosterone. Negative associations were also discovered between some metabolites and sex hormone-binding globulin, semen volume and total sperm count.

Findings are compatible with a weak anti-androgenic action of DEHP metabolites, but less so for DiNP metabolites. Metabolic pathways differed significantly between the three study sites, but without major effect on semen quality or reproductive hormones.
\end{abstract}

(c) 2014 Elsevier Ltd. All rights reserved.
Abbreviations: DEHP, di-2-ethylhexyl phthalate; DiNP, diisononyl phthalate; MEHP, mono-2-ethylhexyl phthalate; MiNP, monoisononyl phthalate; 5OH-MEHP, 2-ethyl-5-hydroxy-hexyl phthalate; 5oxo-MEHP, 2-ethyl-5-oxyhexyl phthalate; 5cx-MEPP, 5-carboxymono-2-ethylpenty phthalate; 7OH-MMeOP, mono-4-methyl-7-hydroxy-octyl phthalate; 7oxo-MMeOP, mono-4-methyl-7-oxo-octyl phthalate; 7cx-MMeOP, mono-4-methyl-7carboxyheptyl phthalate; SHBG, sex hormone-binding globulin; FAI, Free Androgen Index; LH, luteinizing hormone; FSH, follicle stimulation hormone; NAG, Neutral $\alpha$ glucosidase; PSA, Prostate-Specific Antigen; LOD, limit of detection; BMI, body mass index; $\mathrm{CV}$, coefficients of variation; WHO, World Health Organization; PPAR, peroxisome proliferator activated receptors; GLM, general linear models; DMP, dimethyl phthalate; DEP, diethylphthalate; DBP, dibutyl phthalate.

* Corresponding author at: Department of Occupational and Environmental Medicine, Bispebjerg Hospital, University Hospital of Copenhagen, Bispebjerg Bakke 23, DK-2400 Copenhagen NV, Denmark. Tel.: +45 3531 3771; fax: + 4535316070 .

E-mail addresses: ina.olmer.specht@regionh.dk (I.O. Specht), gunntoft@rm.dk (G. Toft), ksh@arbejdsmiljoforskning.dk (K.S. Hougaard), christian.lindh@med.lu.se (C.H. Lindh), V.C.Lenters@uu.nl, d.heederik@uu.nl (V. Lenters), bo_a.jonsson@med.lu.se (B.A.G. Jönsson), aleksander.giwercman@med.lu.se (A. Giwercman),

Jens.Peter.Ellekilde.Bonde@regionh.dk (J.P.E. Bonde).

\section{Introduction}

The hypothalamic-pituitary-gonadal axis and associated hormones are imperative in the regulation of spermatogenesis. Spermatogenesis may consequently be affected by exogenous compounds if they interfere with the endogenous hormone regulation (Gazouli et al., 2002). The ubiquitous phthalates show anti-androgenic action in animal models and in vitro. They therefore represent a class of chemicals that is suspected to harm male fertility in humans (Borch et al., 2006; Christen et al., 2010; Dalsenter et al., 2006). For example, di-2ethylhexyl phthalate (DEHP) exposure in the pre- and post-pubertal male rat decreases serum testosterone levels and adversely affects Leydig cell proliferation in the adult rat testis (Li et al., 2012; Noriega et al., 2009), but DEHP has also been found to antagonize the androgen receptor in vitro (Christen et al., 2010; Shen et al., 2009). 
Phthalates are di-esters of 1,2-benzenedicarboxylic acid (phthalic acid). They have low water solubility, which decreases with increasing length of the side chain and with higher molecular weight (Lyche et al., 2009). The use of phthalates in industrial production mainly depends on their molecular weight. High molecular weight phthalates (ester side-chain lengths with five or more carbons), such as DEHP and diisononyl phthalate (DiNP), are used as plasticizers in numerous polyvinyl chloride products, such as raincoats and footwear, wall coverings and flooring, food packaging, gloves, toys and medical devices (Kavlock et al., 2002a, 2002b; Rock et al., 1986). Low weight phthalates, such as dimethyl phthalate (DMP), diethyl phthalate (DEP) and dibutyl phthalate (DBP), are used as solvents in personal care products, lacquers, insecticides and coatings (Duty et al., 2005a). Humans are mainly exposed to phthalates through ingestion and dermal contact while inhalation is of minor importance outside the occupational setting (Bradley et al., 2013; Wormuth et al., 2006). The compounds or their metabolites have been detected in urine in more than $95 \%$ of the investigated men and women in Western parts of the world (Wittassek et al., 2007).

The biological half-life of phthalates is short and therefore they do not accumulate in the body. A study of a healthy man intentionally ingesting DEHP observed 65-70\% urinary excretion $44 \mathrm{~h}$ after oral intake (Koch et al., 2004). For DiNP, the urinary excretion was $43.6 \%$ after $49 \mathrm{~h}$ (Wittassek and Angerer, 2008). The di-ester phthalates are rapidly hydrolyzed into mono-esters during absorption. The monoesters of the less polar phthalates are further metabolized at different positions in the ester chain by oxidation and hydroxylation into secondary metabolites, which are excreted in the urine as such or after conjugation to glucuronic acid (Hans-Detlev Gilsing et al., 2002; Lyche et al., 2009). The primary metabolite of DEHP, the mono-ester mono-2-ethylhexyl phthalate (MEHP), can be metabolized to, among others, 2-ethyl-5hydroxy-hexyl phthalate (5OH-MEHP) and 2-ethyl-5-oxyhexyl phthalate (50xo-MEHP) or 5-carboxy-mono-2-ethylpenty phthalate (5cx-MEPP) (Fig. 1). In humans, urinary levels of the DEHP secondary metabolites 5OH-MEHP and 50xo-MEHP are several folds higher than the levels of MEHP (Koch et al., 2003). Analysis of these metabolites may therefore increase the analytical sensitivity compared to analysis of MEHP (Koch et al., 2003). Furthermore, the chance of contamination during preparation and analysis of urine samples is greater for MEHP than for the secondary metabolites (Frederiksen et al., 2010; Koch et al., 2003). Finally, most reproductive effects are exerted by the primary- or secondary mono-ester metabolites and not by the di-ester itself (Martino-Andrade and Chahoud, 2010). The metabolism of DiNP is similar to that of DEHP (Fig. 1). Metabolic pathways may impact the toxicity of the phthalate, but only a few studies regarding metabolism and reproductive health exist (Joensen et al., 2012; Meeker et al., 2009).

In most in vitro and rodent studies of phthalate toxicity, the doselevels are orders of magnitude above environmental levels. Interestingly, low-level dose animal studies have found the same anti-androgenic effects on male rat development (Christiansen et al., 2010). It has been questioned whether it is possible to extrapolate animal data to humans. A recent study of xenografted human or rat fetal testis tissue into castrate male mice treated with DBP or monobutyl phthaltate (a metabolite of DBP) found that contrary to the rat, steroidogenesis of the human fetal testis was not suppressed by DBP (Mitchell et al., 2012). In contrast, an in vitro study based on cultured adult human testis tissue showed that testosterone production was inhibited by DEHP and MEHP (Dsdoits-Lethimonier et al., 2012). Validation studies as well as studies of other phthalates are needed, but these findings indicate that research into the effect of DEHP on testicular function is needed.

The number of epidemiologic studies of male reproductive function and environmental and occupational phthalate exposure has increased during the last few years. Several studies indicate that urinary DEHP metabolites may reduce total and free testosterone levels (Joensen et al., 2012; Meeker et al., 2009; Mendiola et al., 2012; Pan et al., 2006), also decreased estradiol and follicle stimulation hormone (FSH) have been reported (Joensen et al., 2012; Meeker et al., 2009). Studies on semen quality have, however, yielded conflicting results. One study investigating DEHP in semen found significantly negative associations with sperm concentration, normal morphology and motility (Pant et al., 2008), but these results have not been replicated in studies investigating DEHP metabolites in other media (Han et al., 2013; Hauser et al., 2006; Herr et al., 2009; Liu et al., 2012; Toshima et al., 2012). Altogether, these results point towards adverse, although inconsistently so, effects of DEHP and DiNP on reproductive function.

Hypothesizing that DEHP and DiNP metabolites exert anti-androgenic effects in humans, the aim of this study was to assess the associations of semen quality and male reproductive hormones relative to serum concentrations of the phthalates. Second, the aim was to investigate whether

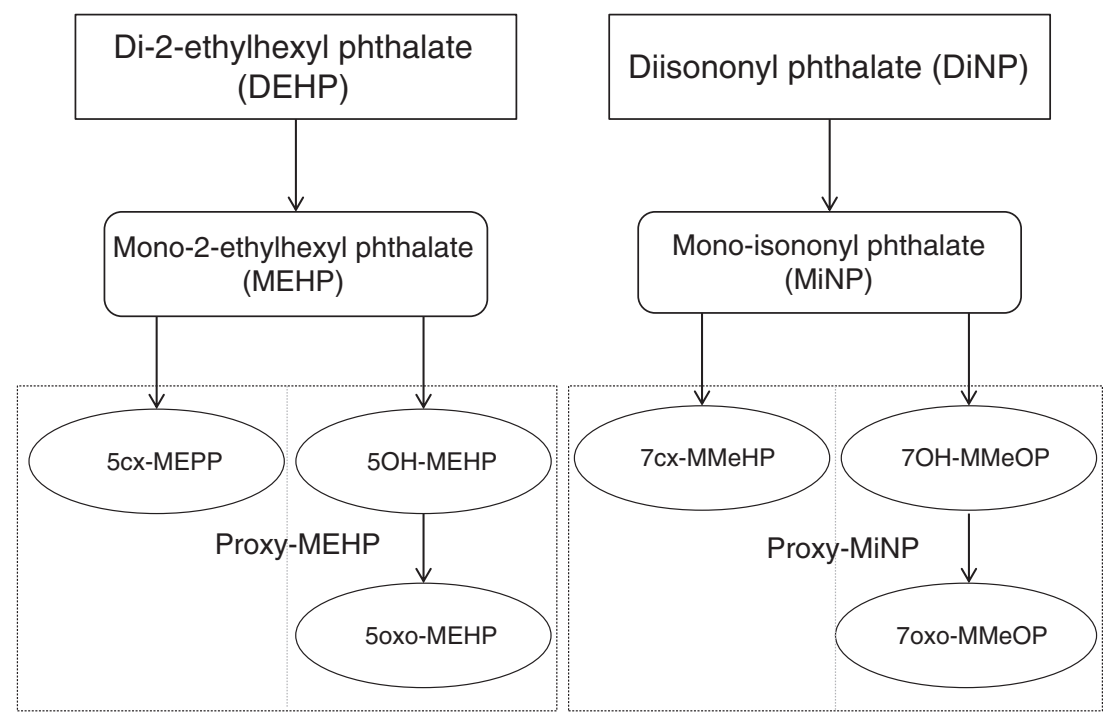

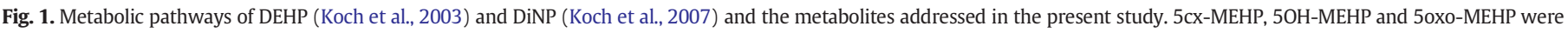

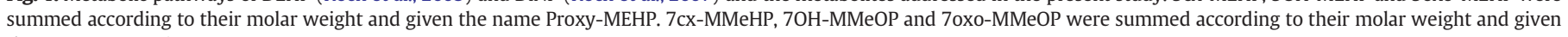
the name Proxy-MiNP. 
the metabolic pathways of DEHP and DiNP influence the effects on semen quality and reproductive hormones.

\section{Material and methods}

\subsection{Study population}

The study population and collection of semen and blood samples have previously been described in detail (Specht et al., 2012; Toft et al., 2005). Briefly, between May 2002 and February 2004 a total of 3833 pregnant women and their male partners were invited consecutively at the first routine antenatal care visit at local hospitals in 19 cities and settlements throughout Greenland, a large central hospital in Warsaw, Poland, and from three hospitals and eight antenatal clinics in Kharkiv, Ukraine.

Both partners were 18 years or more of age and born in the country of the study. In total 1710 couples agreed to participate (44.6\%). Among these male spouses, a study of semen quality and reproductive hormones was formed. The men were enrolled until approximately 200 men in each region had been enlisted. A total of 602 men provided a semen sample, had a venous blood sample drawn and were interviewed regarding lifestyle, occupational and reproductive histories. Thirteen serum samples were lost and were therefore excluded. The final number of subjects with complete information was 589 men.

In spite of a modest participation rate, selection bias is unlikely since the time to pregnancy did not differ between couples where the men provided a semen sample in comparison with couples where the men declined to deliver semen (Specht et al., 2012).

\subsection{Collection and analysis of semen samples}

Prior to the study, all local staff involved in analyzing semen parameters participated in two quality control workshops resulting in inter-observer variability below $11 \%$ for sperm concentration and motility assessment (Toft et al., 2005).

The participants were instructed to collect a semen sample by masturbation at their residence or in privacy in a room at the hospital after a preferred period of sexual abstinence of minimum two days. The sample was kept close to the body to maintain a temperature close to $37^{\circ} \mathrm{C}$ during transport to the laboratory, immediately after collection. After liquefaction, semen samples were analyzed for motility and concentration of spermatozoa by one trained technician at each study site. Briefly, for each sperm sample, the sperm concentration was determined on two aliquots of diluted semen samples (1:10 or 1:20) using an Improved Neubauer Hemacytometer (Paul Marienfeld, Bad Mergentheim, Germany). Sperm cell motility was determined by counting the proportion of $a$ ) fast progressive sperm; $b$ ) slowly progressive sperm; $c$ ) local motile sperm; and $d$ ) immotile sperm on 100 spermatozoa within each of two fresh drops of semen, placed on a preheated $\left(37^{\circ} \mathrm{C}\right)$ clean glass slide covered with a cover slip (Toft et al., 2007). Sperm morphology was determined for all samples at the Fertility Centre, Malmö University Hospital, on Papanicolaou-stained smears. All sperm analyses followed the recommendations by the World Health Organization (WHO) 1999 (Toft et al., 2005).

One hundred and sixteen of the Greenlandic semen samples were collected up to one year after enrollment, where the interview and blood sampling occurred, since the laboratory was not available at the time of enrollment.

The Prostatus $^{\mathrm{TM}}$ kit (Wallac Oy, Finland) was used to determine the concentration of Prostate-Specific Antigen (PSA) in seminal plasma. This immunofluorometric method consists of three monoclonal antibodies against PSA (Elzanaty et al., 2002). A colorimetric method was used to determine the concentration of zinc in seminal plasma (Makino et al., 1982). Trichloroacetic acid was used for the precipitation of the proteins in the sample and then, the supernatant was mixed with a water-soluble pyridylazo dye and the absorbance was measured at $560 \mathrm{~nm}$ (Elzanaty et al., 2002). Seminal plasma Neutral $\alpha$-glucosidase (NAG) was measured using a commercially available kit (Episcreen ${ }^{\circledR}$; Fertipro, Gent, Belgium) according to the instructions given by the manufacturer (Elzanaty et al., 2002). The concentration of fructose in seminal plasma was determined with a spectrophotometric method run on a Beckman Synchron LX20 instrument (Elzanaty et al., 2002). Proteins in the sample were precipitated with perchloric acid and the absorbance of the supernatant was measured. After converting fructose to glucose by addition phosphoglucose isomerase, the absorbance was measured again. The absorbance difference corresponds to the concentration of fructose in the sample (Elzanaty et al., 2002). PSA, zinc, NAG and fructose are markers of epididymal and accessory gland functions.

\subsection{Collection of blood samples}

Venous blood samples were collected within one week of the semen collection for all men, except for 116 of the Greenlandic samples, which were collected up to one year in advance of the semen sample. All sampling devices were ethanol rinsed at Lund University and sent to Greenland, Poland and Ukraine. The blood samples were centrifuged immediately after collection and sera were stored at $-80{ }^{\circ} \mathrm{C}$ for later analysis.

\subsection{Determination of reproductive hormones}

All assays were performed at Malmö University Hospital, Sweden. Serum concentrations of luteinizing hormone (LH), FSH and estradiol were analyzed with immunofluorometric techniques using the UniCel DxI 800 Beckman Access Immunoassay system (Chaska, MN, USA). The total assay coefficients of variation (CV) were $2.6 \%, 2.9 \%$ and $8.1 \%$, respectively, with lower limits of detection (LODs) for the assays of $0.2 \mathrm{IU} / \mathrm{L}, 0.2 \mathrm{IU} / \mathrm{L}$, and $8.0 \mathrm{pmol} / \mathrm{L}$, respectively. Serum testosterone levels were measured by means of a competitive immunoassay (Access; Beckman Coulter Inc., Fullerton, CA, USA) with a LOD of $0.35 \mathrm{nmol} / \mathrm{L}$ and total assay $\mathrm{CV}$ of $2.8 \%$ at $2.9 \mathrm{nmol} / \mathrm{L}$ and $3.2 \%$ at $8.1 \mathrm{nmol} / \mathrm{L}$. Sex hormone-binding globulin (SHBG) concentrations were measured using a fluoro-immunoassay (Immulite 2000; Diagnostic Products Corporation, Los Angeles, CA, USA). The LOD was $0.02 \mathrm{nmol} / \mathrm{L}$ with a total assay CV of $5.5 \%$ and $4.6 \%$, respectively. Inhibin B levels were assessed using a specific immunometric method, as previously described, with a detection limit of $15 \mathrm{ng} / \mathrm{L}$ and intra-assay and total assay CVs < 7\% (Rignell-Hydbom et al., 2005). Free Androgen Index (FAI) was calculated by dividing total testosterone by SHBG, multiplied by 100 . Furthermore, we calculated the ratio between testosterone and LH (T/LH ratio), as a proxy for Leydig cell function.

\subsection{Determination of phthalates}

Phthalate metabolites were analyzed in serum samples by liquid chromatography tandem mass spectrometry at the Department of Occupational and Environmental Medicine at Lund University, Sweden. Analyses included the secondary oxidized metabolites of DEHP (5OH-MEHP, oxo-MEHP and 5cx-MEPP), and DiNP (mono-4-methyl7-hydroxy-octyl phthalate (7OH-MMeOP), mono-4-methyl-7-oxooctyl phthalate (7oxo-MMeOP) and mono-4-methyl-7-carboxyheptyl phthalate (7cx-MMeOP)).

For the analysis of phthalate metabolites, aliquots of $100 \mu \mathrm{L}$ serum were added with isotopically labeled internal standards for all evaluated compounds. The samples were digested with glucuronidase and the proteins were precipitated using acetonitrile. The samples were prepared in 96-well plates and analyzed using a triple quadrupole linear ion trap mass spectrometer (QTRAP 5500; AB Sciex, Foster City, CA, USA) coupled to a liquid chromatography system (UFLCXR, Shimadzu Corporation, Kyoto, Japan; LC/MS/MS) without any further clean-up. The analysis was performed in negative ion mode. All data acquisition 
was performed using Analyst 1.6.1 software and data processing was performed using Multiquant 2.1 (AB Sciex).

The quality of the analyses was checked by including chemical blank samples and an in-house quality control in all analyzed sample batches. Moreover, each sample was analyzed three times in three different analytical batches. The samples were analyzed in randomized order. The imprecision in the analyzed control sample was $8 \%$ for $50 \mathrm{H}-$ MEHP, $9 \%$ for 50xo-MEHP, $18 \%$ for 5cx-MEPP, $8 \%$ for $70 H-M M e O P, 7 \%$ for 7 oxo-MMeOP and $19 \%$ for $7 \mathrm{cx}-\mathrm{MMeOP}$. The LODs were $0.2 \mathrm{ng} / \mathrm{mL}$ for $50 \mathrm{H}$-MEHP, $0.2 \mathrm{ng} / \mathrm{mL}$ for 5oxo-MEHP, $0.1 \mathrm{ng} / \mathrm{mL}$ for $5 \mathrm{cx}-\mathrm{MEPP}$, $0.1 \mathrm{ng} / \mathrm{mL}$ for $7 \mathrm{OH}-\mathrm{MMeOP}, 0.03 \mathrm{ng} / \mathrm{mL}$ for 7oxo-MMeOP and $0.1 \mathrm{ng} / \mathrm{mL}$ for $7 \mathrm{cx}-\mathrm{MMeOP}$. The laboratory is a European reference laboratory for urinary phthalate metabolite analyses for all the included compounds (www.eu-hbm.info/democophes).

All blood samples were taken between 6:30 AM and 4:30 PM. Seventy five percent were taken before 11:00 AM. The time of blood sampling was not related to phthalate concentrations in serum.

\subsection{Statistical analyses}

The total serum concentrations of the DEHP metabolites $(50 \mathrm{H}-$ MEHP, 50xo-MEHP and 5cx-MEPP) as well as the DiNP metabolites (7OH-MMeOP, 7oxo-MMeOP and 7cx-MMeHP) were summarized on a molar basis to provide an estimate of the internal concentration of the mother compounds, DEHP and DiNP, respectively. We named the new variables Proxy-MEHP and Proxy-MiNP, referring to proxy estimates of the DEHP primary metabolite, MEHP, and the DiNP primary metabolite monoisononyl phthalate (MiNP), respectively (Fig. 1).

We limited the regression analyses of single metabolites to phthalates for which $>70 \%$ of the data was above the LOD (Lubin et al., 2004). Two of the six phthalates, 5oxo-MEHP (48.7\% > LOD) and 7oxoMMeOP $(39.2 \%>$ LOD), were for that reason dichotomized by LOD
( $\leq$ LOD and $>$ LOD). Values $<$ LOD for all phthalates were imputed by the maximum likelihood single imputation method (Lubin et al., 2004).

General linear models (GLM) were used to analyze crude and adjusted associations between the exposures (Proxy-MEHP, Proxy-MiNP and serum concentrations of single secondary phthalate metabolites) and outcomes (reproductive hormones and semen quality). Associations were tested across both continuous and categorical levels of phthalates $(<25 \%, 25-75 \%,>75 \%)$.

Residuals were checked for normality and the following continuous variables were transformed by natural logarithm: The four phthalate metabolites, (5OH-MEHP, 5cx-MEPP, 7OH-MMeOP and 7cx-MMeHP), Proxy-MEHP and -MiNP, as well as the outcome variables; volume, sperm count, sperm concentration, normal morphology, motility $(C+D)$, fructose, PSA, NAG, zinc, testosterone, estradiol, inhibin B, FSH, LH, FAI and SHBG. For the linear regression models, the beta coefficient can be interpreted as the percentage change in the dependent variable by one percent increase in the independent variable.

First we analyzed the associations between phthalates, reproductive hormones and semen quality across the three sites of study. For overall statistically significant associations, we then explored the countryspecific associations. It was reasonable to collapse data across the three study sites because of similar distributions of phthalates, hormonal concentrations as well as seminal measures.

In the multiple general linear models investigating semen quality, we adjusted for known and potential confounders, namely, sexual abstinence period ( $\log ($ days $)$ ), age (years), body mass index $\left(B M I, \mathrm{~kg} / \mathrm{m}^{2}\right)$, cotinine in serum $(\log (\mathrm{ng} / \mathrm{mL}))$, spillage of semen sample (yes/no), study site (Greenland, Poland and Ukraine, only in the overall analysis), self-reported genital infections (yes/no) and testicular disorders (yes/no) (as defined in Table 1). In the models of reproductive hormones, we adjusted for age, BMI and study site. When analyzing phthalates according to semen volume and sperm count, observations with spillage of semen samples were excluded ( $\mathrm{n}=65$ excluded). We performed trend

Table 1

Demographic and seminal characteristics of the study populations.

\begin{tabular}{|c|c|c|c|c|c|c|c|c|c|c|c|c|}
\hline & \multicolumn{4}{|c|}{ Greenland ( $\mathrm{N}=196)$} & \multicolumn{4}{|c|}{ Poland ( $\mathrm{N}=190)$} & \multicolumn{4}{|c|}{ Ukraine ( $N=203$ ) } \\
\hline & Percent $^{\mathrm{a}}$ & Mean & Median & Range & Percent $^{\mathrm{a}}$ & Mean & Median & Range & Percent $^{\mathrm{a}}$ & Mean & Median & Range \\
\hline \multicolumn{13}{|l|}{ Demographic characteristics } \\
\hline Age (years) & & 31.0 & 30.6 & $18.5-51.3$ & & 30.3 & 29.6 & $20.4-46.3$ & & 26.5 & 25.0 & $16.2-45.3$ \\
\hline Body mass index $\left(\mathrm{kg} / \mathrm{m}^{2}\right)$ & & 26.1 & 25.5 & $14.0-57.8$ & & 25.8 & 25.4 & $18.5-38.1$ & & 24.2 & 24.0 & $17.7-35.9$ \\
\hline Caffeinated drinks per day & & 6.8 & 6.0 & $0.5-30.0$ & & 4.4 & 4.0 & $1.0-13.0$ & & 3.3 & 3.0 & $1.0-14.0$ \\
\hline Cotinine $(\mathrm{ng} / \mathrm{mL})$ & & 165.1 & 143.5 & $0.4-570.0$ & & 40.0 & 0.4 & $0.4-445.7$ & & 153.5 & 119.7 & $0.4-799.2$ \\
\hline Current smokers & 72.7 & & & & 27.2 & & & & 66.7 & & & \\
\hline$>14$ alcoholic drinks per week & 26.8 & & & & 11.2 & & & & 0.7 & & & \\
\hline Mother smoked during pregnancy & 62.3 & & & & 14.7 & & & & 1.7 & & & \\
\hline Fever in the last three months & 13.3 & & & & 8.7 & & & & 9.9 & & & \\
\hline Currently employed & 85.9 & & & & 87.4 & & & & 76.4 & & & \\
\hline Ever genital infection ${ }^{\mathrm{b}}$ & 82.4 & & & & 5.1 & & & & 4.9 & & & \\
\hline Testicular disorders ${ }^{\mathrm{c}}$ & 0.5 & & & & 4.1 & & & & 3.4 & & & \\
\hline Period of abstinence (days) & & 5.3 & 3.0 & $0.5-240.0$ & & 7.7 & 4.0 & $0.1-90.0$ & & 3.9 & 3.0 & $0.5-11.0$ \\
\hline \multicolumn{13}{|l|}{ Seminal characteristics } \\
\hline Total sperm count $\left(\mathrm{x} 10^{6}\right)$ & & 245.7 & 184.4 & $2.4-1496.0$ & & 345.2 & 197.2 & $3.7-2071.0$ & & 250.4 & 178.9 & $2.1-1442.3$ \\
\hline Concentration (mill/mL) & & 72.0 & 52.6 & $0.6-374.0$ & & 89.2 & 64.0 & $2.1-419.0$ & & 73.3 & 59.4 & $0.4-320.0$ \\
\hline Volume $(\mathrm{g})$ & & 3.5 & 3.2 & $0.7-9.7$ & & 3.8 & 3.5 & $0.5-10.5$ & & 3.5 & 3.1 & $0.7-10.8$ \\
\hline Normal morphology & & 7.0 & 6.0 & $0.0-20.0$ & & 6.7 & 6.0 & $0.0-16.0$ & & 7.4 & 7.0 & $0.0-18.0$ \\
\hline Motility C + D (\%) & & 45.0 & 40.0 & $13.0-99.0$ & & 40.0 & 36.0 & $8.0-100.0$ & & 45.9 & 44.5 & $7.5-100.0$ \\
\hline \multicolumn{13}{|l|}{ Hormonal characteristics } \\
\hline Testosterone $(\mathrm{nmol} / \mathrm{mL})$ & & 14.8 & 14.2 & $3.2-26.9$ & & 13.0 & 12.7 & $4.8-24.0$ & & 18.0 & 17.6 & $5.5-36.5$ \\
\hline $\mathrm{LH}(\mathrm{IU} / \mathrm{L})$ & & 4.4 & 4.1 & $1.4-13.2$ & & 4.1 & 3.7 & $1.3-8.9$ & & 4.2 & 4.0 & $1.3-12.7$ \\
\hline FSH (IU/L) & & 4.9 & 4.3 & $0.0-14.6$ & & 4.0 & 3.6 & $0.7-16.7$ & & 4.2 & 3.4 & $1.0-21.3$ \\
\hline FAI & & 55.3 & 52.4 & $22.3-106.3$ & & 60.7 & 55.9 & $24.3-164.0$ & & 69.8 & 66.5 & $21.0-172.3$ \\
\hline Estradiol (pmol/mL) & & 65.5 & 65.3 & $32.8-112.6$ & & 75.8 & 68.9 & $37.5-297.3$ & & 84.0 & 78.9 & $33.0-159.6$ \\
\hline Inhibin B (ng/L) & & 184.2 & 181.0 & $48.0-470.0$ & & 158.0 & 153.0 & $22.0-338.0$ & & 194.7 & 186.0 & $55.0-390.0$ \\
\hline SHBG (nmol/mL) & & 28.4 & 27.9 & $11.1-55.0$ & & 23.6 & 21.6 & $5.9-63.7$ & & 27.7 & 26.8 & $9.4-64.0$ \\
\hline
\end{tabular}

a Percent if the variable is dichotomous.

b Genital infections: epididymitis, gonorrhea, Chlamydia or mumps in adulthood.

c Testicular disorders: treatment for retracted testis, surgery for varicose veins, torsio testis or testis cancer. 
analyses to investigate dose-effect relationships between phthalates and outcomes. MEHP can be metabolized to either 50H-MEHP and 5oxoMEHP or to 5cx-MEPP, and MiNP can be metabolized to either $70 \mathrm{H}-$ MMeOP and 7oxo-MMeOP or to 7cx-MMeHP (Fig. 1). We performed sub-analyses to investigate whether one of the metabolic pathways was more toxic in regard to semen quality or reproductive hormones.

We made new variables, one for DEHP serum metabolites and one for DiNP serum metabolites, by calculating the ratio between the two metabolic pathways (5OH-MEHP + 5oxoMEHP:5cx-MEPP or 7OHMMeOP + 7oxo-MMeOP:7cx-MMeHP) and afterwards dichotomizing the ratio at the 75 th percentile. We chose to dichotomize at $75 \%$ since we were interested in the extreme values rather than the average. We then investigated associations between the dichotomized ratio and semen quality and reproductive hormones both overall and within each study site.

We checked data for modifying effects of cotinine, smoking status (yes/no), study site, season (winter, spring, summer and fall), BMI, testosterone, and genital infections, the epididymal and accessory gland function (fructose, PSA, NAG, zinc) and the effect of ProxyMEHP/MiNP categories ( $<25 \%, 25-75 \%,>75 \%)$ on semen quality. As a posteriori analysis, based on the finding that phthalates were highest in spring, we also investigated if spring (yes/no) modified the effect of proxies on outcomes.

Statistical analysis was performed with SAS software, version 9.3 for Windows (SAS Institute Inc., Cary, NC, USA).

\subsection{Ethical approval}

The local ethical committees representing all participating populations approved the study and all subjects signed an informed consent.

\section{Results}

The demographic, seminal and hormonal characteristics of the 589 men from Greenland, Poland and Ukraine with complete data on serum concentrations of the six phthalate metabolites are summarized in Table 1 . The men were approximately 30 years of age in Greenland and Poland and 26 years in Ukraine. In Greenland, Poland and Ukraine, $73 \%, 27 \%$ and $67 \%$ of the men, respectively, were smokers.

\subsection{Serum phthalate concentrations}

The serum phthalate concentrations were significantly different at the three study sites for four of the six phthalates (5OH-MEHP, 5oxoMEHP, 5cx-MEPP and 7OH-MMeOP, Table 2). The metabolite with the highest serum concentration was 5cx-MEPP (median levels of 1.1, 1.6 and $1.9 \mathrm{ng} / \mathrm{mL}$ in Greenland, Poland and Ukraine, respectively). Overall, the median concentration of Proxy-MEHP was $0.01 \mathrm{nM}$ and $0.003 \mathrm{nM}$ for Proxy-MiNP. The two proxies were highly correlated $(r=0.79, \mathrm{p}$ $<0.0001$ ).

The phthalate concentrations of 5cx-MEPP, 7oxo-MMeOP and 7cxMMeHP did not differ across seasons, but 5OH-MEHP, 5oxo-MEHP and $7 \mathrm{OH}-\mathrm{MMeOP}$ serum concentrations were highest in spring (data not shown).

\subsection{Associations between phthalates and reproductive hormones}

Table 3 shows the phthalate concentrations divided into three groups based upon quartiles ( $<25 \%, 25-75 \%,>75 \%$ ) or, for phthalates with more than $30 \%<$ LOD, two categories ( $\leq$ LOD, $>$ LOD), together with the corresponding mean values of testosterone, SHBG and T/LH ratio. Only these three outcomes are shown because of significant associations, whereas none were for the other outcomes. Proxy-MEHP and -MiNP were negatively associated with testosterone and, for ProxyMiNP, SHBG. One percent increase in nM Proxy-MEHP yields a $0.07 \%$ decrease in testosterone and one percent increase in Proxy-MiNP yields

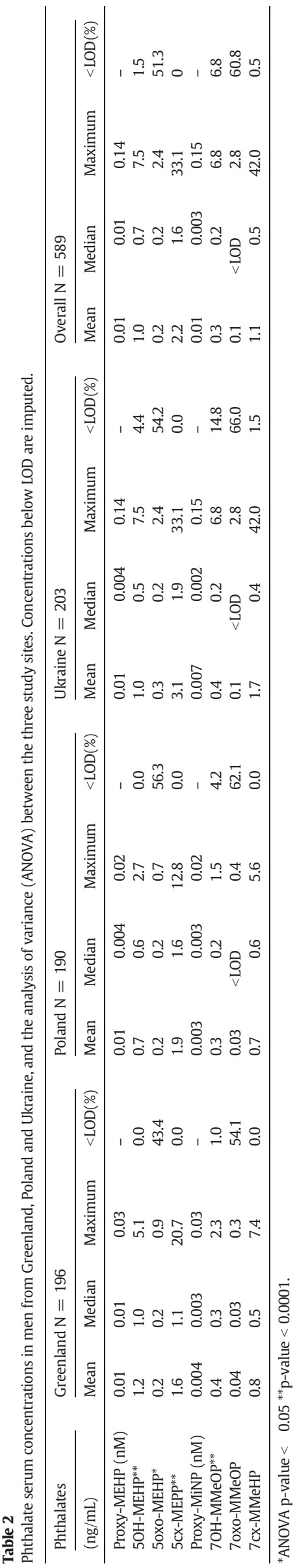


a $0.06 \%$ decrease in both testosterone and SHBG. When Proxy-MEHP or MiNP were divided into groupes, men in the upper quartile of ProxyMEHP or -MiNP exposure groups had $8.4 \%$ and $9.2 \%$ lower serum testosterone, respectively, compared with men in the lowest quartile, with a significant negative trend. Men in the upper Proxy-MiNP quartile group had $9.1 \%$ lower SHBG.

As shown in Table 3, all secondary metabolites, except 5oxo-MEHP, were negatively associated with testosterone in the overall analysis. SHBG was negatively associated with 7cx-MMeHP, and the T/LH ratio was negatively associated with 5OH-MEHP. LH, FSH, estradiol, FAI and inhibin B were not associated with serum phthalate metabolite concentrations (Table 1 in supplementary data).

We did not observe the same significant results when the metabolites were grouped into percentiles as in the linear regression analysis where both the exposure and the outcome were on continuous scales. However, for many associations, significant trends were observed (Table 3).

\subsection{Associations between phthalates and semen quality}

Proxy-MEHP was associated with decreased semen volume and total sperm count, but not with decreased sperm concentration (Table 4 ). Semen volume decreased with $0.09 \%$ per percent increase in Proxy-MEHP, corresponding to a 5.3\% decrease in volume from the highest to the lowest quartile, whereas sperm count decreased with $0.15 \%$, equivalent to a $10.5 \%$ decrease from the highest to the lowest quartile. Proxy-MiNP was not associated with any of the semen parameters (Table 4).
Semen volume and total sperm count decreased with increasing phthalate levels of the secondary metabolite 50H-MEHP (Table 4). A similar pattern was observed for 7OH-MMeOP.

Sperm concentration (Table 4), morphology and sperm motility (data not shown) were not associated with serum phthalate metabolite concentrations.

Furthermore, no interactions between cotinine, smoking status, research site, season, BMI, testosterone, sexual abstinence or genital infections and Proxy-MEHP/MiNP and semen quality were observed (data not shown).

\subsection{Analyses within study sites}

Statistically significant associations found in the overall analysis were investigated within each study site. In Table 5 Proxy-MEHP and -MiNP and the corresponding secondary metabolites were analyzed by study site and are shown for testosterone, SHBG, semen volume, total sperm count and T/LH ratio. For testosterone, SHBG and volume, the associations remained significant and negative in Greenland and/ or Ukraine with negative trends in Poland, except for Proxy-MEHP and volume, and Proxy-MiNP and SHBG. The association with the T/LH ratio was negative in all study sites but only significantly so in Greenland and Poland.

\subsection{Metabolic pathways}

In Fig. 2 the mean levels of the metabolites in each study sites are shown. The dominating metabolic pathways differed significantly

Table 3

Testosterone, SHBG and LH/T ratio according to metabolites of DEHP and DiNP in serum. Metabolites have been divided into $<25 \%, 25-75 \%$, $>75 \%$ or dichotomized by LOD.

\begin{tabular}{|c|c|c|c|c|c|c|c|c|c|c|c|c|}
\hline \multirow[b]{2}{*}{ Metabolites of DEHP } & \multicolumn{4}{|c|}{ Testosterone (nmol/L) } & \multicolumn{4}{|c|}{ SHBG (nmol/L) } & \multicolumn{4}{|c|}{$\mathrm{T} / \mathrm{LH}$ ratio } \\
\hline & $\mathrm{n}$ & Mean & Adj.mean $^{\mathrm{a}}$ & $(95 \% \mathrm{CI})$ & $\mathrm{n}$ & Mean & Adj.mean $^{c}$ & $(95 \% \mathrm{CI})$ & $\mathrm{n}$ & Mean & Adj.mean $^{a}$ & $(95 \% \mathrm{CI})$ \\
\hline \multicolumn{13}{|l|}{ Proxy-MEHP (nM) } \\
\hline $0.002-0.006$ & 102 & 16.1 & $15.4^{\mathrm{b}}$ & $(14.3 ; 16.7)$ & 102 & 28.1 & 26.1 & $(24.8 ; 28.5)$ & 129 & 0.3 & 3.9 & $(4.3 ; 3.5)$ \\
\hline $0.006-0.012$ & 226 & 15.7 & 14.8 & $(14.0 ; 15.7)$ & 225 & 27.0 & 25.2 & $(23.7 ; 26.9)$ & 207 & 0.3 & 3.8 & $(4.2 ; 3.5)$ \\
\hline $0.012-0.124$ & 126 & 15.7 & 14.1 & $(13.1 ; 15.1)$ & 126 & 25.6 & 25.4 & $(21.7 ; 25.2)$ & 118 & 0.3 & 3.4 & $(3.8 ; 3.1)$ \\
\hline \multicolumn{13}{|l|}{ 50H-MEHP (ng/mL) } \\
\hline $0.12-0.44$ & 119 & 17.3 & $15.6^{\mathrm{b}}$ & $(14.5 ; 16.9)$ & 119 & 28.4 & 26.7 & $(24.5 ; 29.1)$ & 119 & 4.9 & $3.8^{\mathrm{b}}$ & $(3.2 ; 4.5)$ \\
\hline $0.44-1.22$ & 214 & 15.3 & 14.7 & $(13.9 ; 15.6)$ & 214 & 25.8 & 24.1 & $(22.7 ; 25.7)$ & 214 & 4.2 & 3.5 & $(3.0 ; 4.1)$ \\
\hline $1.22-7.49$ & 121 & 15.2 & 14.1 & $(13.2 ; 15.2)$ & 120 & 27.2 & 24.8 & $(23.0 ; 26.7)$ & 121 & 3.9 & 3.2 & $(2.7 ; 3.8)$ \\
\hline \multicolumn{13}{|l|}{ 5oxo-MEHP (ng/mL) } \\
\hline$<$ LOD & 228 & 15.3 & 13.9 & $(12.5 ; 15.5)$ & 227 & 26.2 & 25.5 & $(22.7 ; 28.7)$ & 226 & 4.5 & 3.7 & $(3.1 ; 4.3)$ \\
\hline $0.20-2.44$ & 226 & 16.3 & 14.3 & $(12.9 ; 16.0)$ & 226 & 27.5 & 26.1 & $(23.2 ; 29.4)$ & 228 & 4.1 & 3.4 & $(2.9 ; 4.0)$ \\
\hline \multicolumn{13}{|l|}{ 5cx-MEPP (ng/mL) } \\
\hline $0.29-1.01$ & 104 & 15.7 & 14.8 & $(13.7 ; 16.1)$ & 103 & 27.1 & 24.1 & $(22.1 ; 26.3)$ & 104 & 4.2 & 3.6 & $(3.0 ; 4.3)$ \\
\hline $1.01-2.23$ & 224 & 15.9 & 15.1 & $(14.3 ; 16.0)$ & 224 & 27.5 & 25.9 & $(24.3 ; 27.5)$ & 224 & 4.5 & 3.7 & $(3.1 ; 4.3)$ \\
\hline $2.23-33.13$ & 126 & 15.7 & 13.9 & $(12.9 ; 14.9)$ & 126 & 25.6 & 23.4 & $(21.7 ; 25.3)$ & 126 & 4.1 & 3.2 & $(2.7 ; 3.8)$ \\
\hline \multicolumn{13}{|l|}{ Metabolites of DiNP } \\
\hline \multicolumn{13}{|l|}{ Proxy-MiNP (nM) } \\
\hline $0.001-0.002$ & 129 & 17.3 & $15.3^{b}$ & $(14.2 ; 16.4)$ & 129 & 28.2 & 25.5 & $(23.6 ; 27.6)$ & 129 & 4.7 & 3.9 & $(4.3 ; 3.5)$ \\
\hline $0.002-0.004$ & 207 & 15.3 & 14.9 & $(14.0 ; 15.8)$ & 207 & 26.4 & 25.1 & $(23.5 ; 26.7)$ & 207 & 4.2 & 3.8 & $(4.2 ; 3.5)$ \\
\hline $0.004-0.152$ & 118 & 15.0 & 13.9 & $(12.9 ; 14.9)$ & 117 & 26.2 & 23.7 & $(22.0 ; 25.7)$ & 118 & 4.0 & 3.4 & $(3.8 ; 3.1)$ \\
\hline \multicolumn{13}{|l|}{ 7ОH-MMeOP (ng/mL) } \\
\hline $0.04-0.15$ & 122 & 17.9 & $16.0^{\mathrm{b}}$ & $(14.9 ; 17.2)$ & 122 & 28.8 & 26.3 & $(24.4 ; 28.5)$ & 229 & 4.9 & 3.8 & $(3.2 ; 4.5)$ \\
\hline $0.15-0.35$ & 119 & 15.3 & 14.5 & $(13.7 ; 15.4)$ & 229 & 25.7 & 24.3 & $(22.9 ; 25.9)$ & 123 & 4.1 & 3.4 & $(3.0 ; 4.1)$ \\
\hline $0.35-6.81$ & 103 & 14.6 & 13.6 & $(12.6 ; 14.7)$ & 102 & 27.2 & 24.2 & $(22.3 ; 26.3)$ & 103 & 4.0 & 3.4 & $(2.9 ; 4.0)$ \\
\hline \multicolumn{13}{|l|}{ 7oxo-MMeOP (ng/mL) } \\
\hline$<$ LOD & 178 & 15.0 & $13.6^{* c}$ & $(12.2 ; 15.2)$ & 117 & 26.5 & 25.5 & $(22.6 ; 28.7)$ & 276 & 4.5 & $3.6^{*}$ & $(3.1 ; 4.3)$ \\
\hline $0.03-2.82$ & 276 & 16.3 & 15.5 & $(13.0 ; 16.1)$ & 276 & 27.1 & 26.0 & $(23.1 ; 29.2)$ & 178 & 4.0 & 3.3 & $(2.8 ; 3.9)$ \\
\hline \multicolumn{13}{|l|}{ 7cx-MMEHP (ng/mL) } \\
\hline $0.03-0.35$ & 120 & 17.5 & $15.5^{\mathrm{b}}$ & $(14.4 ; 16.7)$ & 120 & 29.1 & $26.1^{\mathrm{b}}$ & $(24.1 ; 28.3)$ & 120 & 4.7 & 3.6 & $(3.0 ; 4.3)$ \\
\hline $0.35-0.86$ & 220 & 15.1 & 14.6 & $(13.8 ; 15.5)$ & 219 & 26.0 & 23.3 & $(23.3 ; 26.4)$ & 220 & 4.2 & 3.7 & $(3.1 ; 4.3)$ \\
\hline $0.86-41.97$ & 114 & 15.4 & 14.1 & $(13.1 ; 15.2)$ & 114 & 26.1 & 21.9 & $(21.9 ; 25.6)$ & 140 & 4.0 & 3.2 & $(2.7 ; 3.8)$ \\
\hline
\end{tabular}

CI $95 \%=95 \%$ confidence interval.

a Adjusted for age, cotinine in serum, BMI, and study site.

b Trend $\mathrm{p}<0.05$

c After adjustment for potential confounders, testosterone was 6\% $(\mathrm{p}=0.041)$ higher for men with 7oxo-MMeOP concentration $<$ LOD.

* $\mathrm{p}<0.05$. 
Table 4

Volume, concentration and total sperm count according to metabolites of DEHP and DiNP in serum. Metabolites have been divided into $<25 \%, 25-75 \%$, $>75 \%$ or dichotomized by LOD.

\begin{tabular}{|c|c|c|c|c|c|c|c|c|c|c|c|c|}
\hline \multirow[b]{2}{*}{ Metabolites of DEHP } & \multicolumn{4}{|c|}{ Volume (g) } & \multicolumn{4}{|c|}{ Concentration (mill/mL) } & \multicolumn{4}{|c|}{ Total sperm count $\left(\mathrm{x} 10^{6}\right)$} \\
\hline & $\mathrm{n}$ & Mean & Adj.mean $^{a}$ & $(95 \% \mathrm{CI})$ & $\mathrm{n}$ & Mean & Adj.mean ${ }^{\mathrm{b}}$ & $(95 \% \mathrm{CI})$ & $\mathrm{n}$ & Mean & Adj.mean $^{a}$ & $(95 \% \mathrm{CI})$ \\
\hline \multicolumn{13}{|l|}{ Proxy-MEHP (nM) } \\
\hline $0.002-0.006$ & 131 & 4.0 & 3.8 & $(3.2 ; 4.5)$ & 146 & 78.5 & 56.5 & $(46.8 ; 83.5)$ & 130 & 323.7 & 182.3 & $(127.8 ; 260.1)$ \\
\hline $0.006-0.012$ & 262 & 3.6 & 3.6 & $(3.1 ; 4.3)$ & 296 & 77.6 & 52.6 & $(45.5 ; 60.9)$ & 262 & 285.6 & 163.6 & $(116.5 ; 229.8)$ \\
\hline $0.012-0.124$ & 131 & 3.6 & 3.6 & $(3.0 ; 4.3)$ & 148 & 78.0 & 48.7 & $(40.7 ; 58.2)$ & 131 & 253.6 & 163.2 & $(114.3 ; 233.1)$ \\
\hline \multicolumn{13}{|l|}{ 5OH-MEHP (ng/mL) } \\
\hline $0.12-0.44$ & 134 & 4.2 & $4.0^{*}$ & $(3.4 ; 4.7)$ & 146 & 78.2 & 53.1 & $(43.6 ; 64.6)$ & 133 & 336.3 & 185.5 & $(130.3 ; 264.1)$ \\
\hline $0.44-1.22$ & 262 & 3.5 & 3.4 & $(2.9 ; 4.1)$ & 296 & 77.7 & 53.2 & $(27.1 ; 61.5)$ & 262 & 279.4 & 162.3 & $(115.4 ; 228.2)$ \\
\hline $1.22-7.49$ & 128 & 3.5 & 3.5 & $(3.0 ; 4.2)$ & 148 & 78.1 & 49.9 & $(41.7 ; 59.7)$ & 128 & 251.6 & 158.6 & $(110.7 ; 227.1)$ \\
\hline \multicolumn{13}{|l|}{ 5oxo-MEHP (ng/mL) } \\
\hline$<$ LOD & 277 & 3.8 & $3.8^{*}$ & $(3.2 ; 4.4)$ & 302 & 79.2 & 51.5 & $(39.4 ; 67.3)$ & 276 & 306.9 & $183.4^{*}$ & $(132.6 ; 256.6)$ \\
\hline $0.20-2.44$ & 247 & 3.6 & 3.4 & $(2.9 ; 4.1)$ & 288 & 76.5 & 46.1 & $(35.3 ; 60.1)$ & 247 & 264.9 & 143.0 & $(101.6 ; 201.3)$ \\
\hline \multicolumn{13}{|l|}{ 5cx-MEPP (ng/mL) } \\
\hline $0.29-1.01$ & 136 & 3.6 & $3.5^{*}$ & $(2.9 ; 4.2)$ & 146 & 73.4 & 49.6 & $(40.8 ; 60.2)$ & 135 & 264.9 & $156.5^{*}$ & $(109.0 ; 224.8)$ \\
\hline $1.01-2.23$ & 260 & 3.8 & 3.9 & $(3.0 ; 4.5)$ & 296 & 82.6 & 56.1 & $(48.6 ; 64.8)$ & 260 & 313.2 & 195.3 & $(140.1 ; 272.1)$ \\
\hline $2.23-33.13$ & 128 & 3.5 & 3.4 & $(2.9 ; 4.1)$ & 148 & 73.1 & 47.1 & $(39.2 ; 56.7)$ & 128 & 257.3 & 140.0 & $(98.2 ; 199.6)$ \\
\hline \multicolumn{13}{|l|}{ Metabolites of DiNP } \\
\hline \multicolumn{13}{|l|}{ Proxy-MiNP (nM) } \\
\hline $0.001-0.002$ & 128 & 3.7 & 3.7 & $(3.1 ; 4.4)$ & 147 & 72.9 & 50.3 & $(37.8 ; 67.1)$ & 128 & 269.3 & 179.9 & $(125.3 ; 258.4)$ \\
\hline $0.002-0.004$ & 266 & 3.7 & 3.6 & $(3.1 ; 4.3)$ & 295 & 82.0 & 48.7 & $(37.4 ; 63.4)$ & 265 & 307.2 & 169.7 & $(121.3 ; 237.4)$ \\
\hline $0.004-0.152$ & 130 & 3.7 & 3.7 & $(3.1 ; 4.4)$ & 148 & 74.8 & 45.5 & $(34.2 ; 60.4)$ & 130 & 263.5 & 158.7 & $(111.0 ; 226.8)$ \\
\hline \multicolumn{13}{|l|}{ 70H-MMeOP (ng/mL) } \\
\hline $0.04-0.15$ & 126 & 3.8 & 3.8 & $(3.2 ; 4.6)$ & 147 & 75.2 & 51.7 & $(43.0 ; 62.2)$ & 126 & 304.4 & 185.7 & $(129.1 ; 267.1)$ \\
\hline $0.15-0.35$ & 263 & 3.7 & 3.7 & $(3.2 ; 4.4)$ & 296 & 80.0 & 53.9 & $(46.6 ; 62.3)$ & 262 & 294.7 & 176.8 & $(125.2 ; 249.8)$ \\
\hline $0.35-6.81$ & 135 & 3.6 & 3.5 & $(3.0 ; 4.1)$ & 148 & 76.6 & 49.3 & $(40.9 ; 59.5)$ & 135 & 256.1 & 155.0 & $(109.9 ; 218.5)$ \\
\hline \multicolumn{13}{|l|}{ 7охо-MMeOP (ng/mL) } \\
\hline$<$ LOD & 313 & 3.6 & 3.6 & $(3.1 ; 4.3)$ & 357 & 78.5 & 48.6 & $(37.4 ; 63.2)$ & 312 & 294.0 & 171.2 & $(123.0 ; 238.2)$ \\
\hline $0.03-2.82$ & 211 & 3.8 & 3.7 & $(3.2 ; 4.4)$ & 233 & 77.1 & 48.7 & $(37.0 ; 64.0)$ & 211 & 276.8 & 166.4 & $(118.6 ; 233.4)$ \\
\hline \multicolumn{13}{|l|}{ 7cx-MMEHP (ng/mL) } \\
\hline $0.03-0.35$ & 129 & 3.5 & $3.4^{*}$ & $(2.9 ; 4.0)$ & 147 & 70.9 & 52.8 & $(43.9 ; 63.7)$ & 129 & 243.6 & 162.0 & $(113.6 ; 231.0)$ \\
\hline $0.35-0.86$ & 264 & 3.8 & 3.9 & $(3.3 ; 4.6)$ & 296 & 82.3 & 52.2 & $(45.1 ; 60.3)$ & 263 & 319.0 & 178.5 & $(127.0 ; 251.1)$ \\
\hline $0.86-41.97$ & 131 & 3.6 & 3.6 & $(3.0 ; 4.3)$ & 148 & 76.1 & 51.8 & $(43.1 ; 62.2)$ & 131 & 265.7 & 164.1 & $(115.3 ; 233.8)$ \\
\hline
\end{tabular}

$95 \% \mathrm{CI}=95 \%$ confidence interval.

a Adjusted for age, cotinine in serum, sexual abstinence time, BMI, genital infections, testicular disorder and study site.

b Adjusted for age, cotinine in serum, sexual abstinence time, spillage during sampling, BMI, genital infections, testicular disorder and study site.

$* \mathrm{p}<0.05$.

between the three study sites $(\mathrm{p}<0.001$, in the three study sites the proportion of ratios $>75 \%$ for the two MEHP pathways were 1.3, 2.8 and 3.7, and for the two MiNP pathways: 2.7, 3.4 and 7.1 in Greenland, Poland and Ukraine, respectively), indicating a higher degree of metabolism in 5cx-MEHP and 7cx-MMeHP pathways, especially for men from Ukraine. In the overall analysis, we did not observe differences between men metabolizing MEHP to 5OH-MEHP and 50xo-MEHP or to $5 \mathrm{cx}-$ MEHP, or MiNP to 7OH-MMeOP and 7oxo-MMeOP or to 7cx-MMeHP with respect to semen quality and reproductive hormones (data not shown). When the study sites were analyzed separately, we found that men from Greenland that metabolized DiNP to 7cx-MMeHP in a higher degree than to $70 \mathrm{H}$ - and 7oxo-MMeOP had $1.28 \%((1.05 ; 1.55)$, $\mathrm{p}=0.01$ ) fewer morphologically normal spermatozoa. We also found that men from Poland that metabolized DEHP to $5 \mathrm{cx}-\mathrm{MEPP}$ in a higher degree than to 5OH- and 5oxo-MEHP had $1.22 \mathrm{pmol} / \mathrm{mL}((1.45 ; 1.03)$ $\mathrm{p}=0.02$ ) more estradiol in their serum.

\section{Discussion}

In this cross-sectional study of 589 male partners of pregnant women from Greenland, Poland and Ukraine, we summed serum levels of DEHP and DiNP secondary metabolites on a molar basis to obtain a proxy for internal exposure to the mother compounds (Hans-Detlev Gilsing et al., 2002; Koch et al., 2003). We observed inverse associations between serum levels of Proxy-MEHP and Proxy-MiNP metabolites and the serum concentrations of testosterone and SHBG, and semen volume. The inverse associations between Proxy-MEHP and -MiNP and testosterone were consistent across study sites, although not statistically significant within each site. Often the associations were not significant in
Ukraine. Sperm concentration, morphology and motility, and FAI, LH, FSH, estradiol, inhibin B, NAG, fructose, zinc and PSA were not associated with these phthalate metabolites.

Phthalate exposure is usually determined by measuring the levels of phthalate metabolites in urine. In this study phthalate exposure was measured in serum. There are however some disadvantages with the use of serum instead of urine. Firstly, it has been stated that the primary metabolites in serum are not stable (Koch et al., 2004). The serum samples were frozen rapidly after collection and were subsequently stored at $-80{ }^{\circ} \mathrm{C}$. Secondly, the metabolite levels in serum are much lower than in urine which sets special demands on the used method. With our method we had levels above the LOD for the hydroxy and carboxy metabolites of both DEHP and DiNP in almost all of the samples. Moreover, the precisions were acceptably high. Thirdly, if the serum sample post-collection becomes contaminated with the ubiquitous di-esters of phthalate the lipase activity of serum will split the di-esters into mono-esters. The concentrations of mono-esters in serum are therefore not reliable measures of exposure, contrary to urine that has no lipase activity (Hauser, 2008). Therefore, we did not investigate the monoesters MEHP and MiNP or any other phthalate mono-esters.

Our method for analysis of phthalates has been further validated by correlating the concentrations of the phthalates and their metabolites in serum and urine samples from a group of young Swedish men (Lindh et al., unpublished). The urinary and serum levels of most phthalate metabolites were highly correlated, especially for 5cx-MEPP, 7oxo-MMeOP and $7 \mathrm{cx}-\mathrm{MMeHP}\left(\mathrm{r}_{\mathrm{s}}=0.77-0.91\right)$, indicating that serum and urinary levels can be used as biomarkers for human exposure. For 5OH-MEHP there seemed to be two different groupings, as some men presented with low serum levels compared to the urinary levels and others with 
higher serum levels compared to urinary levels. These observations were not due to post-collection contamination (Lindh et al., unpublished). Lindh et al. proposed that this grouping might reflect differences in metabolic pathways, but this needs to be further investigated. For $7 \mathrm{OH}-\mathrm{MMeOP}$, use of the quantifier ion at $\mathrm{m} / \mathrm{z} 307.2 \rightarrow 121.1$ seemed to be affected by co-elution in the chromatogram from another compound with similar retention-time. The correlation between serum and urine concentrations was much higher when the use of the quantifier ion was changed to $\mathrm{m} / \mathrm{z} 307.2 \rightarrow 77.1$. The sensitivity for this fragment is however much lower and was incompatible with the equipment applied in the present study. Therefore, the associations in the present study between semen volume or testosterone and $7 \mathrm{OH}-$ $\mathrm{MMeOP}$ might be influenced by contamination and warrant further investigation. 50xo-MEHP correlated poorly between serum and urine $\left(r_{S}=0.32\right)$, which might be due to low detection frequency in serum. We took this into account in the statistical analysis, by dichotomizing 50xo-MEHP at the LOD.

Previously it has been observed that phthalate concentrations in urine differed during the day (Meeker et al., 2009). Seventy five percent of the blood samples were taken before 11:00 AM and the remaining 25\% between 11:00 AM and 4:30 PM. In our study the time of blood sampling did not modify phthalate concentrations in serum. However, we observed a seasonal difference in phthalate serum levels for $5 \mathrm{OH}-$ MEHP, 5oxo-MEHP and 7OH-MMeOP, with the highest phthalate levels occurring in spring. This may have caused misclassification toward the null hypothesis in the Greenlandic men, because we found no seasonal variation in semen and hormonal parameters, and blood and semen samples were not sampled concomitantly for 116 out of the 196 men from Greenland.

Population studies worldwide have consistently detected phthalate exposure in more than 95\% of the participants (Wittassek and Angerer, 2008; Wittassek et al., 2007). A German biomonitoring study has shown a downward trend in urinary DEHP and increasing DiNP over a time period of 9 years (1988-2003) (Wittassek et al., 2007). These observations were confirmed in a Danish study of human amniotic fluid from 1980 to 1996 (Jensen et al., 2012). Even considering these long-term trends in DEHP and DiNP, and their short half-life, we believe that the widespread detection is likely to indicate constant (over a couple years) exposure to the two phthalates, and therefore, that for the 116 Inuit men whose semen was not sampled at the same time as blood likely had only a minimal effect on exposure-response estimates.

Serum concentrations of phthalates and levels of reproductive hormones were analyzed at one laboratory, and collection of semen and blood samples was performed according to uniform protocols by one trained professional in each country, to reduce inter-laboratory variations (Toft et al., 2005).

It is generally considered that phthalates exert their action by inhibiting Leydig cell synthesis of testosterone, but in vitro studies have shown both androgenic and anti-androgenic effects at the receptor level, are dependent on the concentration and mixture of the metabolites (Akingbemi et al., 2004; Christen et al., 2010, 2012; Kruger et al., 2008; Sun et al., 2012). Our results are compatible with both mechanisms. We found decreased T/LH ratio with increased 5OH-MEHP exposure, which might indicate an anti-androgenic effect at the testis level because of poorer Leydig cell function with higher 5OH-MEHP levels. At high levels of exposure, the phthalates may act as peroxisome proliferators in rodents (Gazouli et al., 2002; Latini et al., 2008). Phthalates might stimulate the peroxisome proliferator activated receptors (PPARs) in Leydig cells (Gazouli et al., 2002; Latini et al., 2008; Zhang et al., 2008). However, the reproductive toxicity of phthalates cannot be entirely explained by the action of a PPAR $\alpha$-mediated pathway, because PPAR $\alpha$-null mice remain sensitive to DEHP-mediated reproductive toxicity, suggesting the involvement of additional pathways (Zhang et al., 2008).

An in vitro study of the anti-androgenic activity of mixture activities of BBP, DBP, DEP and bisphenol A in MDA-kb2 cells found both a concentration dependent agonistic and antagonistic action on the androgen receptor (Christen et al., 2012). At low mixture concentrations, an androgenic activity occurred (pointing to an agonistic action), yet a strong anti-androgenic activity (antagonistic action) occurred at high concentrations (Christen et al., 2012). This might also apply for DiNP since we observed decreased testosterone as well as SHBG, although this remains unknown. Furthermore, the associations between SHBG, testosterone and Proxy-MiNP could also be driven by the phthalates inhibiting the production of SHBG or testosterone or both. We observed lower testosterone levels but not consistently reduced SHBG with increasing serum levels for all phthalate metabolites or within each study site, which implies that the testicles rather than the liver were affected. Phthalates may also affect the testosterone production in testes without measurable effects on semen parameters since there were no effects on other reproductive hormones involved in the testosterone synthesis, like LH and FSH, although we did see a non-significant decrease in LH for Proxy-MiNP.

Our results cannot be directly compared with other studies of phthalates and male reproductive health, because the panel of exposure measures differed and the phthalates were measured in serum. Urinary levels of the mono-ester MEHP, the most frequently measured DEHP metabolite, may not adequately reflect internal dose. This is because high molecular weight mono-ester phthalates, like MEHP, are further

Table 5

Statistically significant associations from overall analyses analyzed by study site. The adjusted ${ }^{\mathrm{a}} \mathrm{B}(95 \% \mathrm{CI})$ are logarithmic transformed

\begin{tabular}{|c|c|c|c|c|c|}
\hline & & Greenland, ß (95\% CI) & Poland, ß (95\% CI) & Ukraine, ß (95\% CI) & Overall, ß (95\% CI) \\
\hline \multirow[t]{3}{*}{ Volume (g) } & Proxy-MEHP & $0.01(-0.12 ; 0.14)$ & $-0.05(-0.23 ; 0.12)$ & $-0.15(-0.24 ;-0.05)^{*}$ & $-0.09(-0.15 ;-0.02)^{*}$ \\
\hline & 5ОH-MEHP & $-0.02(-0.14 ; 0.11)$ & $-0.06(-0.21 ; 0.05)$ & $-0.11(-0.19 ;-0.03)^{*}$ & $-0.09(-0.15 ;-0.02)^{*}$ \\
\hline & 7ОН-ММеОР & $-0.04(-0.16 ; 0.07)$ & $-0.03(-0.17 ; 0.11)$ & $-0.10(-0.17 ;-0.02)^{*}$ & $-0.06(-0.12 ;-0.00)^{*}$ \\
\hline \multirow[t]{2}{*}{ Total sperm count $\left(\times 10^{6}\right)$} & Proxy-MEHP & $-0.01(-0.30 ; 0.28)$ & $-0.53(-0.98 ;-0.08)^{*}$ & $-0.11(-0.27 ; 0.05)$ & $-0.15(-0.23 ;-0.01)^{*}$ \\
\hline & 5ОН-МЕHР & $0.04(-0.20 ; 0.29)$ & $-0.36(-0.69 ;-0.04)^{*}$ & $-0.15(-0.31 ; 0.02)$ & $-0.13(-0.25 ;-0.00)^{*}$ \\
\hline \multirow[t]{6}{*}{ Testosterone (nmol/L) } & Proxy-MEHP & $-0.11(-0.21 ;-0.01)^{*}$ & $-0.02(-0.19 ; 0.15)$ & $-0.06(-0.12 ; 0.01)$ & $-0.07(-0.12 ;-0.02)^{*}$ \\
\hline & 5ОН-МЕНР & $-0.12(-0.22 ;-0.02)^{*}$ & $-0.10(-0.25 ; 0.07)$ & $-0.03(-0.08 ; 0.02)$ & $-0.04(-0.09 ;-0.02)^{*}$ \\
\hline & 5cx-MEPP & $-0.06(-0.15 ; 0.02)$ & $0.03(-0.13 ; 0.18)$ & $-0.07(-0.12 ;-0.01)^{*}$ & $-0.05(-0.09 ;-0.04)^{*}$ \\
\hline & Proxy-MiNP & $-0.13(-0.24 ;-0.03)^{*}$ & $-0.13(-0.27 ; 0.03)$ & $-0.04(-0.08 ;-0.002)^{*}$ & $-0.06(-0.10 ;-0.02)^{*}$ \\
\hline & 7ОH-MMeoP & $-0.15(-0.24 ;-0.06)^{*}$ & $-0.08(-0.20 ; 0.05)$ & $-0.05(-0.10 ;-0.01)^{*}$ & $-0.07(-0.12 ;-0.03)^{* *}$ \\
\hline & 7cx-MMeHP & $-0.08(-0.16 ; 0.01)$ & $-0.09(-0.23 ; 0.05)$ & $0.03(-0.06: 0.004)$ & $-0.04(-0.07 ;-0.01)^{*}$ \\
\hline \multirow[t]{2}{*}{ SHBG (nmol/L) } & Proxy-MiNP & $0.03(-0.07 ; 0.12)$ & $-0.16(-0.34 ; 0.02)$ & $-0.05(-0.10 ;-0.01)^{*}$ & $-0.06(-0.10 ;-0.01)^{*}$ \\
\hline & 7cx-MMeHP & $0.03(-0.05 ; 0.11)$ & $-0.17(-0.34 ; 0.01)$ & $-0.05(-0.09 ;-0.01)^{*}$ & $-0.04(-0.07 ;-0.004)^{*}$ \\
\hline $\mathrm{T} / \mathrm{LH}$ ratio & 5OH-MEHP & $-0.15(-0.30 ;-0.01)^{*}$ & $-0.27(-0.47 ;-0.07)^{*}$ & $-0.03(-0.10 ; 0.04)$ & $-0.07(-0.14 ;-0.01)^{*}$ \\
\hline
\end{tabular}

$95 \% \mathrm{CI}=95 \%$ confidence interval.

* $\mathrm{p}<0.05^{* *} \mathrm{p}<0.0001$

a Adjusted as indicated in Tables 3 and 4 study site. 


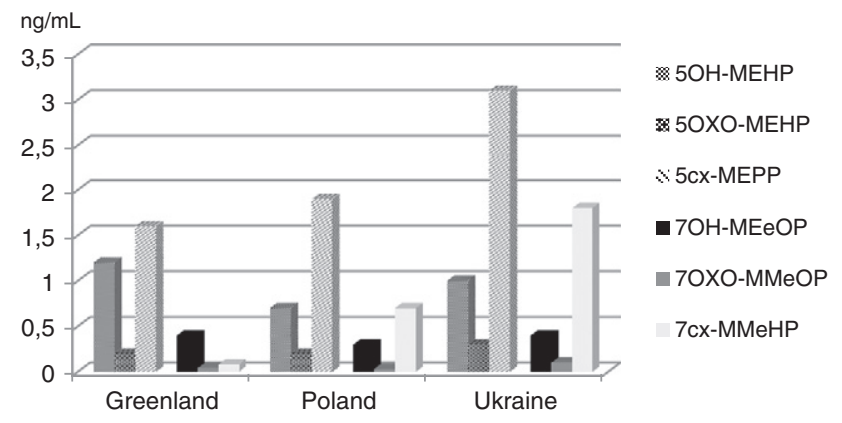

Fig. 2. Mean concentrations of DEHP and DiNP metabolites illustrating metabolic pathways in the three populations. 5OH-MEHP and 5oxo-MEHP belong to the same DEHP pathway and were summed in the analysis. 7OHMEeOP and 7oxo-MMeOP belong to the same DiNP pathway and were summed in the analysis.

metabolized to oxidative products, which are found in higher levels in the urine than MEHP. Therefore the oxidized MEHP metabolites may provide greater analytical sensitivity than MEHP (Frederiksen et al., 2010; Wittassek and Angerer, 2008). The majority of epidemiologic studies of DEHP or DiNP exposure and male fertility have measured phthalate metabolites in urine (Duty et al., 2003, 2005b; Hauser et al., 2006, 2007; Herr et al., 2009; Joensen et al., 2012; Jonsson et al., 2005; Liu et al., 2012; Mendiola et al., 2012); three studies investigating the same secondary DEHP metabolites as we did (Herr et al., 2009; Meeker et al., 2009; Mendiola et al., 2012), and only one study investigated the DiNP metabolites (Joensen et al., 2012). Furthermore, most studies of reproductive function and MEHP in urine have been performed in populations of subfertile couples (Duty et al., 2005b; Hauser et al., 2006, 2007; Herr et al., 2009; Liu et al., 2012; Meeker et al., 2009), although studies have also been undertaken among proven fertile men (Mendiola et al., 2011), in men from the general population (Joensen et al., 2012; Jonsson et al., 2005) and in men with occupational phthalate exposure (Huang et al., 2011; Pan et al., 2006).

Free testosterone and FAI (an indicator of free testosterone (Vermeulen et al., 1999)) have previously been found to decrease with increasing urinary MEHP levels in epidemiologic studies (Joensen et al., 2012; Meeker et al., 2009; Mendiola et al., 2012; Pan et al., 2006). Only a few studies have observed significant associations between total testosterone and MEHP or \%MEHP (Joensen et al., 2012; Meeker et al., 2009), although insignificant decreases in testosterone have been reported (Han et al., 2013; Meeker et al., 2009; Mendiola et al., 2011; Park et al., 2010). Furthermore, MEHP has been associated with decreased estradiol (Meeker et al., 2009) and FSH (Joensen et al., 2012), and increased SHBG (Joensen et al., 2012; Mendiola et al., 2012). Likewise, DiNP has been associated with increased SHBG and decreased FAI (Joensen et al., 2012). These results, observed across different designs and study populations, indicate adverse effects of DEHP and DiNP metabolites on the hypothalamic-pituitary-gonadal axis, especially in regard to free and total testosterone. In a large pooled study of 783 men, Mendiola et al. (2012) found that SHBG was positively associated with the two secondary metabolites of DEHP; 5OH-MEHP and 50xo-MEHP. These, as well as MEHP, were inversely associated with FAI and free testosterone, but not with total testosterone (Mendiola et al., 2012). A primary increase in plasma SHBG levels is associated with lower levels of free testosterone, as SHBG is the major specific binding protein for testosterone (Ruokonen et al., 1985). Thus the associations found between FAI and DEHP might be driven by changes in SHBG (Mendiola et al., 2011). In our study we found both decreased total testosterone and decreased SHBG with increasing levels of Proxy-MiNP, but not Proxy-MEHP. These results do not match those of Mendiola et al. We did not find associations between the phthalate metabolites and FAI, which indicate that Proxy-MiNP might act in an androgenic way, and not anti-androgenic as expected. The diminished
SHBG which leads to lower total testosterone without changes in FAI and $\mathrm{LH}$ is an indication of androgenic action.

Herr et al. (2009) summed MEHP, 5OH-MEHP, 5oxo-MEHP and 5cxMEPP (DEHP-4) and analyzed associations with sperm concentration, motility and morphology. As in our study, they did not find any associations with these measures of semen quality (Herr et al., 2009), although MEHP has been associated with reduced motility of spermatozoa (Huang et al., 2011). Total sperm count and semen volume were negatively associated with 5cx-MEPP in our study, but not with sperm concentration, indicating that the association of total sperm count could be driven by semen volume since total sperm count is calculated by multiplying volume and concentration. From a biological point of view the sperm production in testis, which corresponds to the total sperm count, is however not regulated by the volume. If the effect we observed was solely on the volume, the total sperm count would be unchanged with an increased concentration. We found no indication that this association was confounded or modified by BMI, testosterone level, genital infections, smoking status, sexual abstinence or accessory gland function.

Metabolic pathways of MEHP and MiNP in relation to semen quality and reproductive hormones have, to our knowledge, not previously been investigated in a similar way, although two studies have investigated the proportion of DEHP and DiNP metabolites in the urine measured as MEHP (MEHP\%) and MiNP (\%MiNP), which are considered phenotypic markers of less efficient metabolism of the mother compound to its oxidized metabolites (Joensen et al., 2012; Mendiola et al., 2012). Since we were not able to measure the primary metabolites, our results cannot be compared with results of \%MEHP or \%MiNP. We found no indications that variations in the phthalate mono-ester metabolizing characteristics as measured by the ratio between the two metabolic pathways of the mono-ester were related to semen quality and reproductive hormones in the overall analysis. Thus (genetic) differences in metabolism may not affect the toxicity in fertile men. However, we did observe significant differences between pathways of metabolism in the three study sites, and therefore we analyzed the metabolic pathways separately for each site. Men from Greenland that metabolized DiNP to 7cx-MMeHP in a higher degree than to 7OH- and 7oxo-MMeOP presented with fewer normal spermatozoa, and men from Poland that metabolized DEHP to 5cx-MEPP in a higher degree than to $5 \mathrm{OH}$ - and 40xo-MEHP had a little more estradiol in their serum. These differences were small and possibly chance findings, but differences in metabolism might play a role in phthalate toxicity and ought to be investigated further.

\section{Conclusions}

Overall, we found that increasing exposure to Proxy-MEHP and some metabolites of DEHP were associated with decreased testosterone levels, semen volume and total sperm count. These findings are indicative of an anti-androgenic action of DEHP. Proxy-MiNP and some DiNP metabolites were found to decrease serum testosterone and SHBG, indicating an androgenic effect of DiNP. Metabolic pathways differed significantly between the three study sites, but without major effect on semen quality or reproductive hormones.

\section{Conflict of interest statement}

The authors have no financial interest or other conflicts of interest in the publication of these results.

Supplementary data to this article can be found online at http://dx. doi.org/10.1016/j.envint.2014.02.002.

\section{Acknowledgments}

The study was part of the CLEAR Project: Climate, Environmental Contaminants and Reproductive Health (www.inuendo.dk/clear) 
supported by the European Commission 7th Framework Programme FP7-ENV-2008-1 Environment (including Climate Change) Grant no.: 226217; the Danish Research Council (10-082745) and the ReproSund and ReproHigh projects.Åsa Amilon, Margareta Maxe and Agneta Kristensen are acknowledged for invaluable help with the phthalate metabolite analyses.

\section{References}

Akingbemi BT, Ge R, Klinefelter GR, Zirkin BR, Hardy MP. Phthalate-induced Leydig cell hyperplasia is associated with multiple endocrine disturbances. Proc Natl Acad Sci U S A 2004:101:775-80. [20-1].

Borch J, Metzdorff SB, Vinggaard AM, Brokken L, Dalgaard M. Mechanisms underlying the anti-androgenic effects of diethylhexyl phthalate in fetal rat testis. Toxicology 2006;223:144-55. [1-6].

Bradley EL, Burden RA, Bentayeb K, Driffield M, Harmer N, Mortimer DN, et al. Exposure to phthalic acid, phthalate diesters and phthalate monoesters from foodstuffs: UK total diet study results. Food Addit Contam Part A Chem Anal Control Expo Risk Assess 2013;30(4):735-42.

Christen V, Crettaz P, Oberli-Schrammli A, Fent K. Some flame retardants and the antimicrobials triclosan and triclocarban enhance the androgenic activity in vitro. Chemosphere 2010;81:1245-52.

Christen V, Crettaz P, Oberli-Schrammli A, Fent K. Antiandrogenic activity of phthalate mixtures: validity of concentration addition. Toxicol Appl Pharmacol 2012;259: 169-76. [1-3].

Christiansen S, Boberg J, Axelstad M, Dalgaard M, Vinggaard AM, Metzdorff SB, et al. Low-dose perinatal exposure to di(2-ethylhexyl) phthalate induces anti-androgenic effects in male rats. Reprod Toxicol 2010;30:313-21.

Dalsenter PR, Santana GM, Grande SW, Andrade AJ, Araujo SL. Phthalate affect the reproductive function and sexual behavior of male Wistar rats. Hum Exp Toxicol 2006;25: 297-303.

Dsdoits-Lethimonier C, Albert O, Le BB, Perdu E, Zalko D, Courant F, et al. Human testis steroidogenesis is inhibited by phthalates. Hum Reprod 2012;27:1451-9.

Duty SM, Silva MJ, Barr DB, Brock JW, Ryan L, Chen Z, et al. Phthalate exposure and human semen parameters. Epidemiology 2003;14:269-77.

Duty SM, Calafat AM, Silva MJ, Ryan L, Hauser R. Phthalate exposure and reproductive hormones in adult men. Hum Reprod 2005a;20:604-10.

Duty SM, Ackerman RM, Calafat AM, Hauser R. Personal care product use predicts urinary concentrations of some phthalate monoesters. Environ Health Perspect 2005b;113: 1530-5.

Elzanaty S, Richthoff J, Malm J, Giwercman A. The impact of epididymal and accessory sex gland function on sperm motility. Hum Reprod 2002;17:2904-11.

Frederiksen $\mathrm{H}$, Jorgensen N. Andersson AM. Correlations between phthalate metabolites in urine, serum, and seminal plasma from young Danish men determined by isotope dilution liquid chromatography tandem mass spectrometry. J Anal Toxicol 2010;34: 400-10.

Gazouli M, Yao ZX, Boujrad N, Corton JC, Culty M, Papadopoulos V. Effect of peroxisome proliferators on Leydig cell peripheral-type benzodiazepine receptor gene expression, hormone-stimulated cholesterol transport, and steroidogenesis: role of the peroxisome proliferator-activator receptor alpha. Endocrinology 2002;143:2571-83.

Gilsing Hans-Detlev, Angerer Jürgen, Prescher Dietrich. Monophthalates with oxidized C5-carbon in the ester chain: a simple synthetic access to two major metabolites of bis-(2-ethylhexyl)-phthalate. Monatsh Chem 2002;133:1147-55. [Ref Type: Journal (Full)].

Han X, Cui Z, Zhou N, Ma M, Li L, Li Y, et al. Urinary phthalate metabolites and male reproductive function parameters in Chongqing general population, China. Int J Hyg Environ Health 2014;217(2-3):271-8. http://dx.doi.org/10.1016/j.ijheh.2013.06.006.

Hauser R. Urinary phthalate metabolites and semen quality: a review of a potential biomarker of susceptibility. Int J Androl 2008;31:112-7.

Hauser R, Meeker JD, Duty S, Silva MJ, Calafat AM. Altered semen quality in relation to urinary concentrations of phthalate monoester and oxidative metabolites. Epidemiology 2006; 17:682-91.

Hauser R, Meeker JD, Singh NP, Silva MJ, Ryan L, Duty S, et al. DNA damage in human sperm is related to urinary levels of phthalate monoester and oxidative metabolites. Hum Reprod 2007;22:688-95.

Herr C, Zur AN, Koch HM, Schuppe HC, Fieber C, Angerer J, et al. Urinary di(2-ethylhexyl) phthalate (DEHP) - metabolites and male human markers of reproductive function Int J Hyg Environ Health 2009;212:648-53.

Huang LP, Lee CC, Hsu PC, Shih TS. The association between semen quality in workers and the concentration of di(2-ethylhexyl) phthalate in polyvinyl chloride pellet plant air. Fertil Steril 2011;96:90-4

Jensen MS, Norgaard-Pedersen B, Toft G, Hougaard DM, Bonde JP, Cohen A, et al. Phthalates and perfluorooctanesulfonic acid in human amniotic fluid: temporal trends and timing of amniocentesis in pregnancy. Environ Health Perspect 2012;120:897-903.

Joensen UN, Frederiksen H, Jensen MB, Lauritsen MP, Olesen IA, Lassen TH, et al. Phthalate excretion pattern and testicular function: a study of 881 healthy Danish men. Environ Health Perspect 2012;120:1397-403.

Jonsson BA, Richthoff J, Rylander L, Giwercman A, Hagmar L. Urinary phthalate metabolite and biomarkers of reproductive function in young men. Epidemiology 2005;16:487-93.

Kavlock R, Boekelheide K, Chapin R, Cunningham M, Faustman E, Foster P, et al. NTP Center for the Evaluation of Risks to Human Reproduction: phthalates expert panel report on the reproductive and developmental toxicity of di(2-ethylhexyl) phthalate. Reprod Toxicol 2002a;16:529-653.
Kavlock R, Boekelheide K, Chapin R, Cunningham M, Faustman E, Foster P, et al. NTP Center for the Evaluation of Risks to Human Reproduction: phthalates expert panel report on the reproductive and developmental toxicity of di-isononyl phthalate. Reprod Toxicol 2002b;16:679-708.

Koch HM, Rossbach B, Drexler H, Angerer J. Internal exposure of the general population to DEHP and other phthalates-determination of secondary and primary phthalate monoester metabolites in urine. Environ Res 2003;93:177-85.

Koch HM, Bolt HM, Angerer J. Di(2-ethylhexyl)phthalate (DEHP) metabolites in human urine and serum after a single oral dose of deuterium-labelled DEHP. Arch Toxicol 2004;78:123-30

Koch HM, Muller J, Angerer J. Determination of secondary, oxidised di-iso-nonylphthalate (DINP) metabolites in human urine representative for the exposure to commercial DINP plasticizers. J Chromatogr B Analyt Technol Biomed Life Sci 2007 Mar 1;847(2):114-25.

Kruger T, Long M, Bonefeld-Jorgensen EC. Plastic components affect the activation of the aryl hydrocarbon and the androgen receptor. Toxicology 2008;246:112-23. [18-4].

Latini G, Scoditti E, Verrotti A, De CF, Massaro M. Peroxisome proliferator-activated receptors as mediators of phthalate-induced effects in the male and female reproductive tract: epidemiological and experimental evidence. PPAR Res 2008;2008:359267.

Li XW, Liang Y, Su Y, Deng H, Li XH, Guo J, et al. Adverse effects of di-(2-ethylhexyl) phthalate on Leydig cell regeneration in the adult rat testis. Toxicol Lett 2012;215: 84-91. [30-11].

Lindh Christian H, Virissa Lenters, Jonatan Axelsson, Aleksander Giwercman, Lars Rylander, Gunnar Toft, et al. Determination of perfluorinated compounds, oxidized phthalate metabolites, bisphenol A, cotinine and 25 hydroxy vitamin $\mathrm{D}_{3}$ in small volumes of serum (unpublished).

Liu L, Bao H, Liu F, Zhang J, Shen H. Phthalates exposure of Chinese reproductive age couples and its effect on male semen quality, a primary study. Environ Int 2012;42: 78-83. http://dx.doi.org/10.1016/j.envint.2011.04.005.

Lubin JH, Colt JS, Camann D, Davis S, Cerhan JR, Severson RK, et al. Epidemiologic evaluation of measurement data in the presence of detection limits. Environ Health Perspect 2004;112:1691-6.

Lyche JL, Gutleb AC, Bergman A, Eriksen GS, Murk AJ, Ropstad E, et al. Reproductive and developmental toxicity of phthalates. J Toxicol Environ Health B Crit Rev 2009;12: 225-49.

Makino T, Saito M, Horiguchi D, Kina K. A highly sensitive colorimetric determination of serum zinc using water-soluble pyridylazo dye. Clin Chim Acta 1982;120:127-35. [26-3].

Martino-Andrade AJ, Chahoud I. Reproductive toxicity of phthalate esters. Mol Nutr Food Res 2010;54:148-57.

Meeker JD, Calafat AM, Hauser R. Urinary metabolites of di(2-ethylhexyl) phthalate are associated with decreased steroid hormone levels in adult men. J Androl 2009;30: 287-97.

Mendiola J, Jorgensen N, Andersson AM, Calafat AM, Silva MJ, Redmon JB, et al. Associations between urinary metabolites of di(2-ethylhexyl) phthalate and reproductive hormones in fertile men. Int J Androl 2011;34:369-78.

Mendiola J, Meeker JD, Jorgensen N, Andersson AM, Liu F, Calafat AM, et al. Urinary concentrations of di(2-ethylhexyl) phthalate metabolites and serum reproductive hormones: pooled analysis of fertile and infertile men. J Androl 2012;33: 488-98.

Mitchell RT, Childs AJ, Anderson RA, van den Driesche S, Saunders PT, McKinnell C, et al. Do phthalates affect steroidogenesis by the human fetal testis? Exposure of human fetal testis xenografts to di-n-butyl phthalate. J Clin Endocrinol Metab 2012;97: E341-8.

Noriega NC, Howdeshell KL, Furr J, Lambright CR, Wilson VS, Gray Jr LE. Pubertal administration of DEHP delays puberty, suppresses testosterone production, and inhibits reproductive tract development in male Sprague-Dawley and Long-Evans rats. Toxicol Sci 2009;111:163-78.

Pan G, Hanaoka T, Yoshimura M, Zhang S, Wang P, Tsukino H, et al. Decreased serum free testosterone in workers exposed to high levels of di-n-butyl phthalate (DBP) and di-2-ethylhexyl phthalate (DEHP): a cross-sectional study in China. Environ Health Perspect 2006;114:1643-8.

Pant N, Shukla M, Kumar PD, Shukla Y, Mathur N, Kumar GY, et al. Correlation of phthalate exposures with semen quality. Toxicol Appl Pharmacol 2008;231:112-6. [15-8].

Park MS, Yang YJ, Hong YP, Kim SY, Lee YP. Assessment of di (2-ethylhexyl) phthalate exposure by urinary metabolites as a function of sampling time. J Prev Med Public Health 2010;43:301-8.

Rignell-Hydbom A, Rylander L, Giwercman A, Jonsson BA, Lindh C, Eleuteri P, et al. Exposure to PCBs and p, p'-DDE and human sperm chromatin integrity. Environ Health Perspect 2005;113:175-9.

Rock G, Labow RS, Tocchi M. Distribution of di(2-ethylhexyl) phthalate and products in blood and blood components. Environ Health Perspect 1986;65:309-16.

Ruokonen A, Alen M, Bolton N, Vihko R. Response of serum testosterone and its precursor steroids, SHBG and CBG to anabolic steroid and testosterone self-administration in man. J Steroid Biochem 1985;23:33-8.

Shen O, Du G, Sun H, Wu W, Jiang Y, Song L, et al. Comparison of in vitro hormone activities of selected phthalates using reporter gene assays. Toxicol Lett 2009;191:9-14. [1-12].

Specht IO, Hougaard KS, Spano M, Bizzaro D, Manicardi GC, Lindh CH, et al. Sperm DNA integrity in relation to exposure to environmental perfluoroalkyl substances-a study of spouses of pregnant women in three geographical regions. Reprod Toxicol 2012;33:577-83

Sun H, Si C, Bian Q, Chen X, Chen L, Wang X. Developing in vitro reporter gene assays to assess the hormone receptor activities of chemicals frequently detected in drinking water. J Appl Toxicol 2012;32:635-41. 
Toft G, Axmon A, Giwercman A, Thulstrup AM, Rignell-Hydbom A, Pedersen HS, et al. Fertility in four regions spanning large contrasts in serum levels of widespread persistent organochlorines: a cross-sectional study. Environ Health 2005;4:26.

Toft G, Long M, Kruger T, Hjelmborg PS, Bonde JP, Rignell-Hydbom A, et al. Semen quality in relation to xenohormone and dioxin-like serum activity among Inuits and three European populations. Environ Health Perspect 2007;115(Suppl. 1):15-20.

Toshima H, Suzuki Y, Imai K, Yoshinaga J, Shiraishi H, Mizumoto Y, et al. Endocrine disrupting chemicals in urine of Japanese male partners of subfertile couples: a pilot study on exposure and semen quality. Int J Hyg Environ Health 2012;215:502-6.

Vermeulen A, Verdonck L, Kaufman JM. A critical evaluation of simple methods for the estimation of free testosterone in serum. J Clin Endocrinol Metab 1999;84:3666-72.

Wittassek M, Angerer J. Phthalates: metabolism and exposure. Int J Androl 2008;31: 131-8.
Wittassek M, Wiesmuller GA, Koch HM, Eckard R, Dobler L, Muller J, et al. Internal phthalate exposure over the last two decades-a retrospective human biomonitoring study. Int J Hyg Environ Health 2007;210:319-33.

World Health Organization (WHO). Association WH, WHO Laboratory Manual for the Examination of Human Semen and Sperm-Cervical Mucus interaction. Cambridge New York., Cambridge University Press; 1999.

Wormuth M, Scheringer M, Vollenweider M, Hungerbuhler K. What are the sources of exposure to eight frequently used phthalic acid esters in Europeans? Risk Anal 2006;26:803-24

Zhang Y, Ge R, Hardy MP. Androgen-forming stem Leydig cells: identification, function and therapeutic potential. Dis Markers 2008;24:277-86. 